\title{
NASA Centennial Challenge: 3D-Printed Habitat
}

Tracie Prater, Ph.D.

Materials and Processes

Laboratory NASA Marshall Space

Flight Center

Monsi Roman,

Program Manager, Centennial Challenges

Tony Kim, Deputy Program Manager, Centennial Challenges

Rob Mueller, Ph.D.

Senior Technologist, Swampworks, Kennedy Space Center 


\section{centennialchallenges}

\section{Competition Vision}

- Advance the automated manufacturing and materials technologies needed for fabrication of habitats on a planetary surface using indigenous materials and mission recyclables

- Terrestrially, these technologies stand to revolutionize the construction industry by automating labor intensive processes and enabling rapid fabrication of large scale structures

- World's population will increase from 6.6 billion to 12.9 billion by 2100

- Requires aggressive construction practices to satisfy increased demand for housing

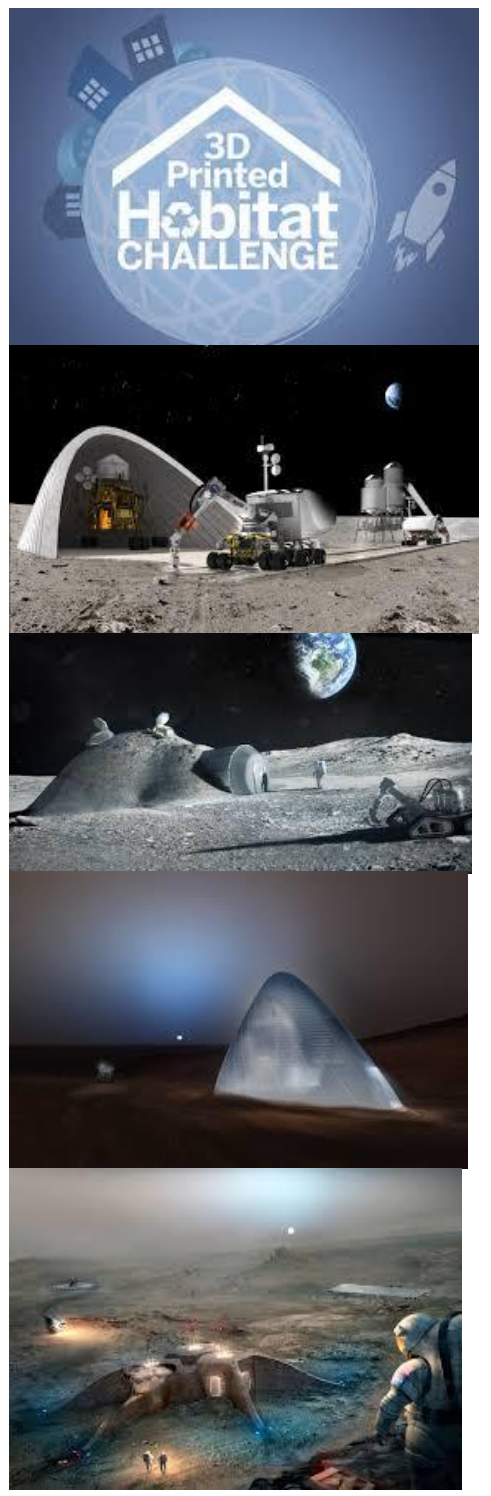




\section{centennialchallenges}

\section{What is 3D Printing?}

- Process of constructing a 3D object by depositing material layer by layer based on a digital part file

\section{Why use 3D Printing for construction?}

- Removes design constraints ("manufacturing for design")

- Enables building and testing earlier in project lifecycle

- Ability to work with new material formulations

- Maximize use of in situ resources (planetary surface

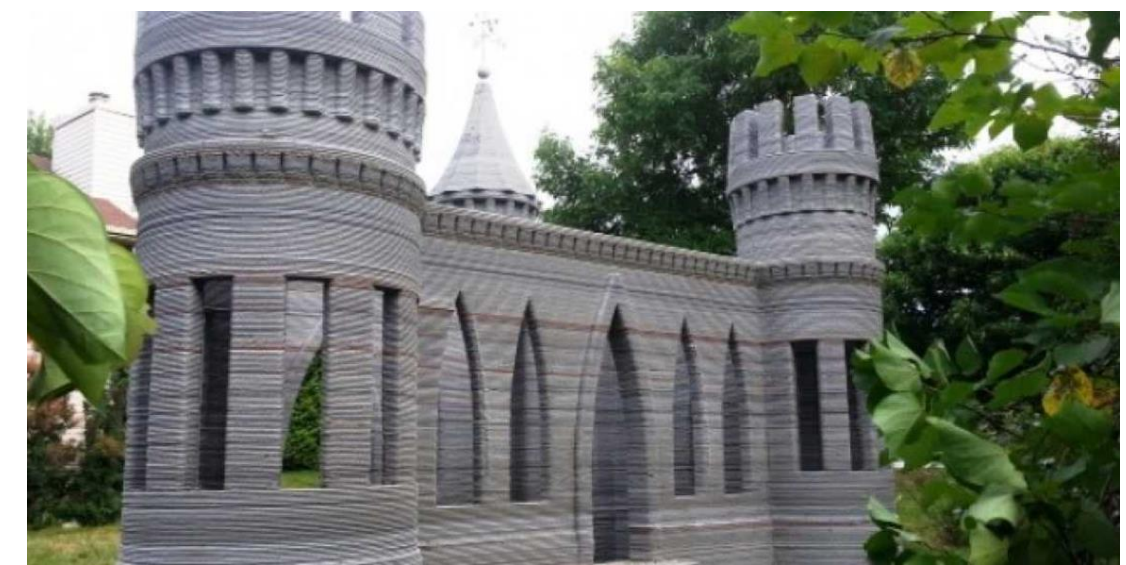

3D printed concrete castle construction applications) 


\section{centennialchallenges}

\section{Overview of current approaches}

- In general, processes may be:

- Contour Crafting process (USC)

- Cement based materials extruded through a nozzle

- Process used for NASA/Army Corps of Engineers project Additive Construction for Mobile Emplacement (ACME)

- Fused Deposition Modeling

- Material extruded in wire form

- Same process used by many desktop printers

- In general, printing systems may be:

- Gantry style systems

- Extruder is attached to frame that translates in the $x-y$ plane

- 6 degree of freedom robotic systems

- Extruder is the end effector of an industrial robot

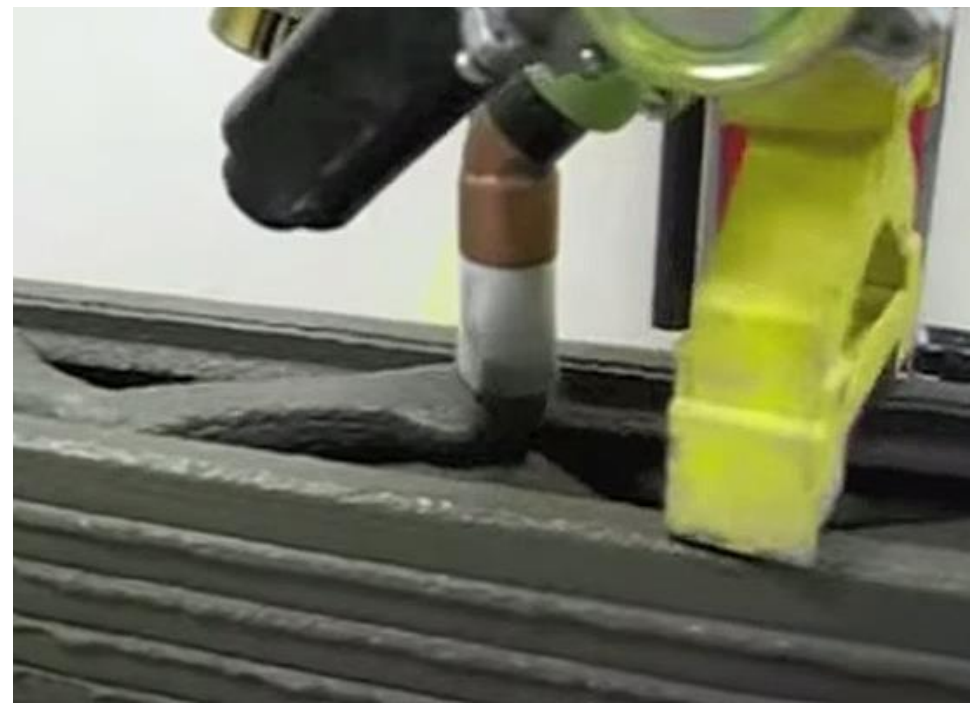

Image of concrete extrusion process from Contour Crafting 


\section{centennialchallenges}

\section{Potential of 3D Printing Technologies for Space and Earth}

- Autonomous systems can fabricate infrastructure (potentially from indigenous materials) on precursor missions

- Can serve as a key enabling technology for exploration by reducing logistics (i.e. launch mass) and eliminating the need for crew tending of manufacturing systems

- Also has potential to address housing needs in light of unprecedented population growth

- Disaster response

- Military field operations

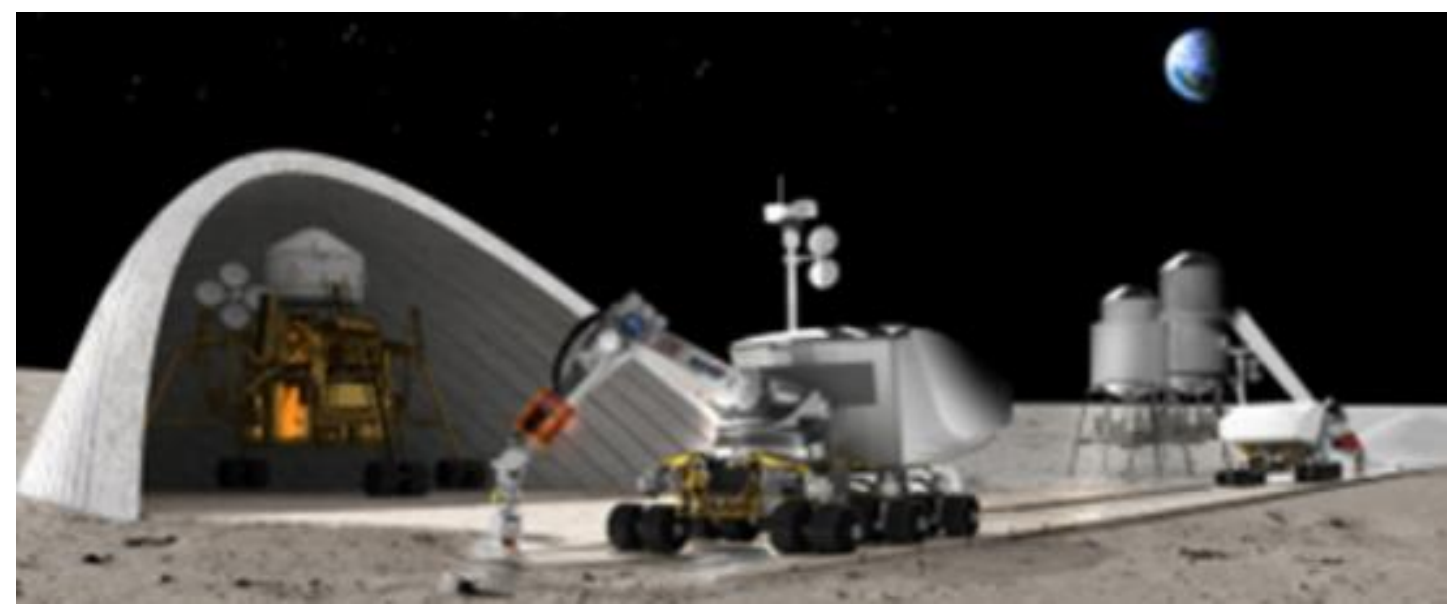

Artist's rendering of

manufacturing

operations on a

planetary surface 


\section{centennialchallenges}

\section{Centennial Challenge: 3D Printed Habitat}

Objective: Advance additive construction technology needed to create sustainable housing solutions for Earth and beyond

\section{Autonomous, Sustainable Additive Manufacturing of Habitats}

\section{Phase 1}

\section{Design:}

Develop state-of-the-art architectural concepts that take advantage of the unique capabilities offered by 3D printing.

Prize Purse Awarded: $\$ 0.04 \mathrm{M}$

\section{Phase 2}

Structural Member:

Demonstrate an additive manufacturing material system to create structural components using terrestrial/space based materials and recyclables.

Prize Purse: $\$ 1.1 \mathrm{M}$
Phase 3

\section{On-Site Habitat:}

Building on material technology progress from Phase 2, demonstrate an automated to build a full-scale habitat.

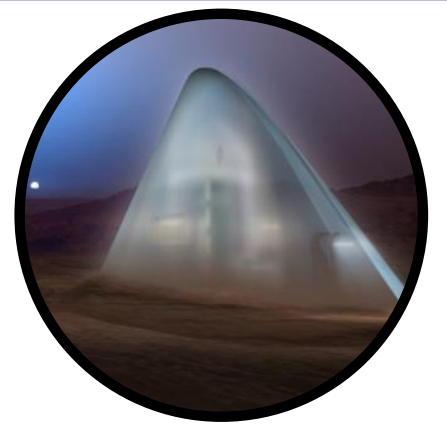

Mars Ice House, winner of the Phase I competition from Space

Exploration Architecture and Clouds AO 


\section{centennialchallenges}

\section{D Printed Habitat: Phase II Competition}
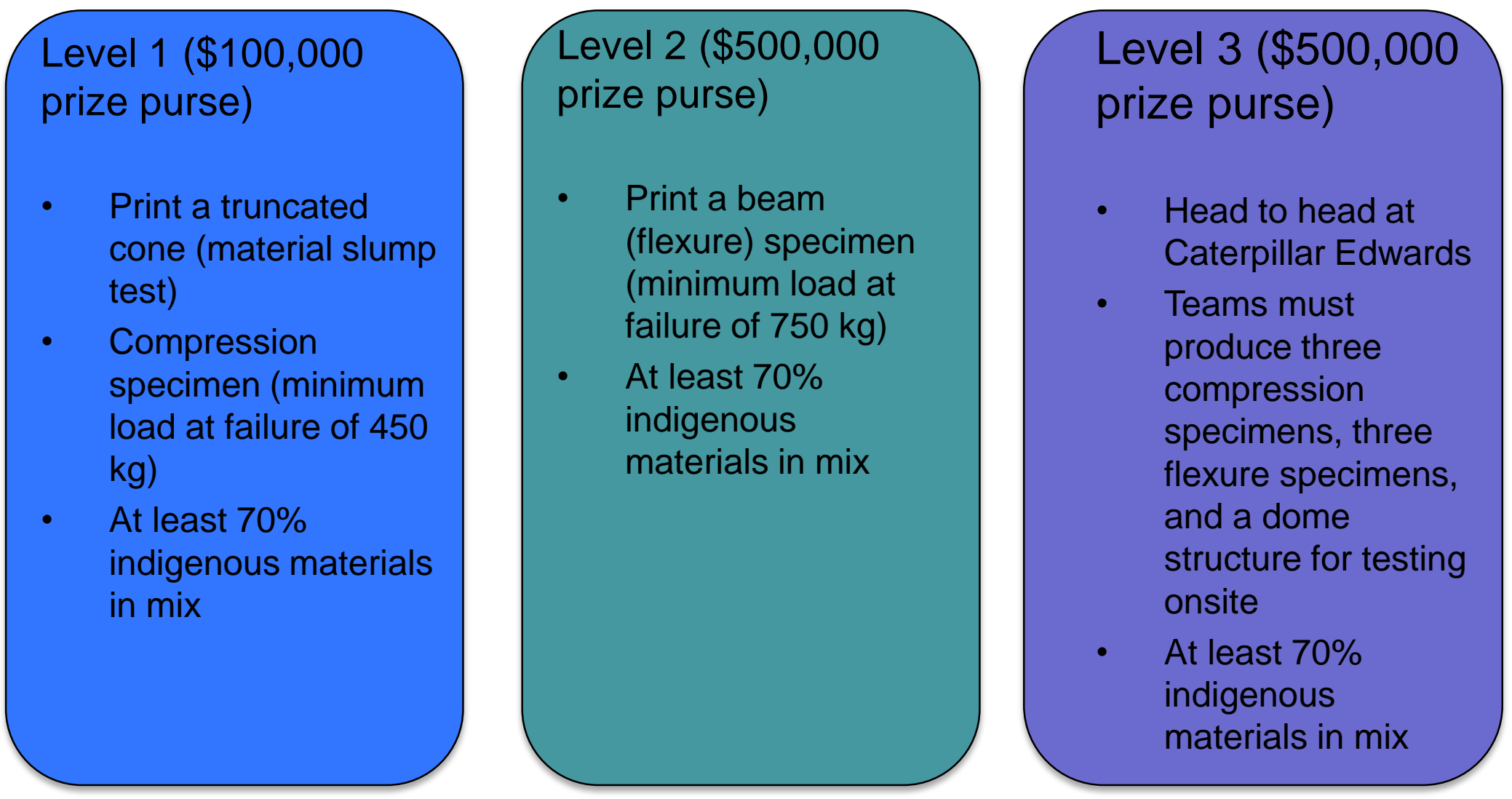

Phase II is run by Bradley University with Caterpillar as the primary sponsor. Additional sponsors include Bechtel Construction Company and Brick \& Mortar Ventures. 


\section{centennialchallenges}

\section{Challenge Roles}

\section{Centennial Challenges (CC): Program Office}

Challenge Role: Oversee the execution of the Challenge and ensure that the outcomes meet the overall goals of NASA and the Centennial Challenges program office

\section{Bradley University (BU): Allied Organization}

501(c)(3) nonprofit University with comprehensive array of undergraduate and graduate academic programs in business, communications, education, engineering, fine arts, health sciences, liberal arts and sciences, and technology.

- Challenge Role: Conduct 3D Printed Habitat Challenge in partnership with Caterpillar by control and maintain the rules, organize the judging process, coordinate with judges'schedules, direct incremental levels, logistics, and receive data submittals. AO will also coordinate the registration process.

\section{Caterpillar (CAT): Challenge Main Sponsor}

Technical Challenge Facilitator

- Private company specializing heavy construction vehicles and machinery

- Providing facilities, logistics, and capability to host head-to-head competition

- Challenge Role: Assist Bradley in designing the Technical Details of the Challenge. Engage the Technical Communities that can participate as Challenge Competitors, and amplify the Challenge message to the broader Open Innovation Community

- Connects organizations with external innovation resources to accelerate innovation in private, public and social sectors 


\section{centennialchallenges}

\section{Challenge Roles}

\section{Marshall Space Flight Center (MSFC): Technology Lead}

Challenge Role: Subject Matter Experts; Design the Challenge; define desired technology and transition path

\section{Kennedy Space Center (KSC): Technology support}

Challenge Role: Subject Matter Experts; Design the Challenge; define desired technology and transition path

\section{Bechtel:}

Challenge Role: Private Company supporting the rules development and potential benefactor for successful competitors. "This type of construction challenge that has been essence of the Bechtel Corporation's more than one hundred years of history. Participating in meeting and overcoming the challenges of inter-planetary construction will help to ensure that our organization will remain an industry leader for the next one hundred years."

\section{Brick \& Mortar:}

Challenge Role: Private Company supporting the rules development and potential benefactor for successful competitors. 


\section{centennialchallenges}

\section{Timeline for All Phases}

\section{Phase 1}

Completed

Sept 2015
Phase 2

Registration

Closes

Jan 31, 2017
Phase 2

Level 1

Submission

Deadline

Mar 31, 2017
Phase 2

Level 3

Competition

Aug 24-27,

2017

\section{Phase 2}

Registration

Opens

Oct 1, 2016
Phase 2

Level 2

Submission

Deadline

May 31, 2017

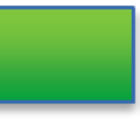

2018

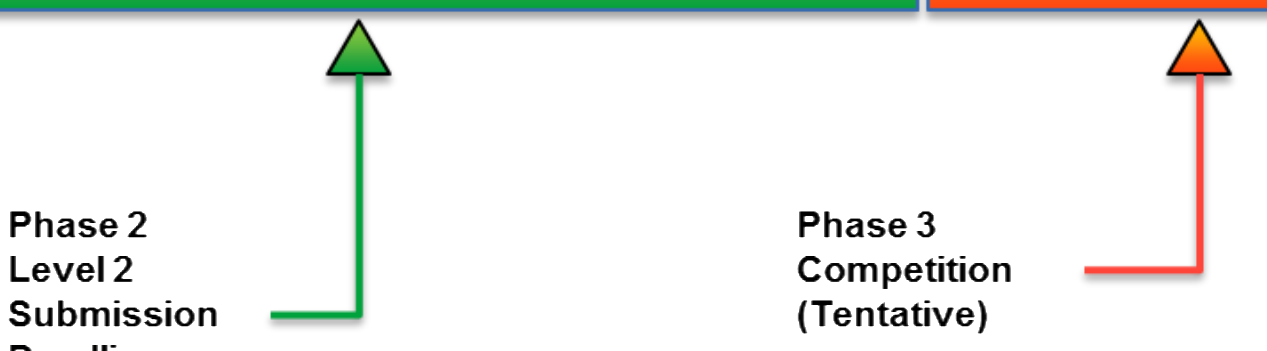




\section{centennialchallenges}

\section{Phase II Competition: Materials}

- One objective of competition is creation of construction materials from indigenous materials and mission waste (polymer recyclables which would otherwise be "nuisance" materials)

- Sliding materials scale rates material based on relevance to planetary missions

- Teams are penalized for use of imported materials (those that would be transported to earth specifically for construction purposes)

- Polymer scale is based on frequency of use of polymeric materials in packaging for the International Space Station (ISS)

- Aggregate scale is based on relative availability of materials on the planetary surface

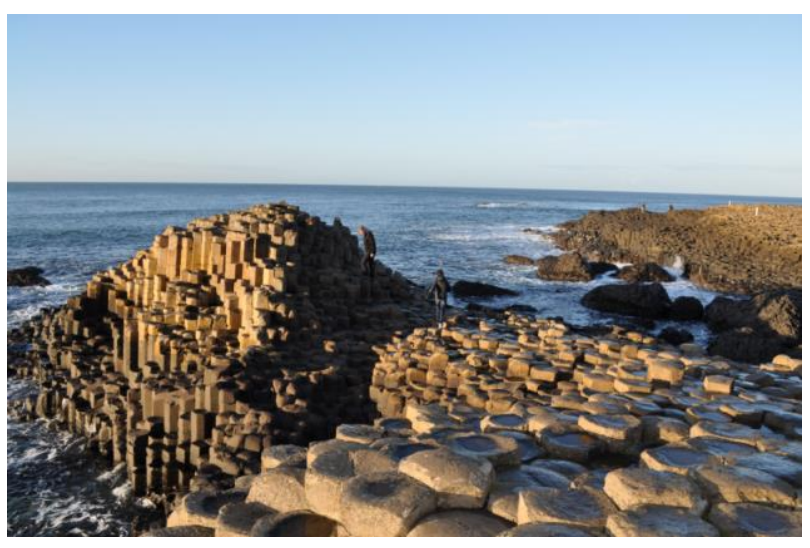

Basalt, considered an indigenous material, is rated highly on the sliding scale for phase II 


\section{centennialchallenges}

\section{Phase II Competition: Materials}

\begin{tabular}{|c|c|}
\hline $\begin{array}{l}\text { Material } \\
\text { Applicability }\end{array}$ & Options \\
\hline $\begin{array}{l}\text { Aggregate/ } \\
\text { Indigenous }\end{array}$ & $\begin{array}{l}\mathrm{CBI} \text { - } \quad \text { Crushed basaltic igneous rock ( } \mathrm{SiO} 2 \text { weight percent less than or equal to } \\
\text { 57) } \\
\text { BSR - Basaltic sedimentary rocks (talus, alluvium with very little } \\
\text { alteration/weathering, or mine tailings) } \\
\text { GSS - Gypsum sand and siliceous sedimentary rocks (e.g., sand box sand, } \\
\text { mudstone) } \\
\text { CSR - Carbonaceous sedimentary rocks (e.g., limestone, dolomite) } \\
\text { IRS - } \quad \text { Igneous rocks with } \mathrm{SiO} 2 \text { weight percent greater than } 57 \text { (e.g., granite) } \\
\text { MR - } \quad \text { Metamorphic rocks (e.g., slate) }\end{array}$ \\
\hline Recyclables & $\begin{array}{ll}\text { LP - } & \text { Low Density Polyethylene (\#4 recycle code) } \\
\text { HP - } & \text { High Density Polyethylene (\#2 recycle code) } \\
\text { PT - } & \text { Polyethylene Terephthalate (\#1 recycle code) } \\
\text { NY - } & \text { Nylon (\#7 recycle code) } \\
\text { PP - } & \text { Polypropylene (\#5 recycle code) } \\
\text { AF - } & \text { Aluminum Foil or ground up aluminum parts } \\
\text { PS - } & \text { Polystyrene (\#6 recycle code) } \\
\text { VY - } & \text { Vinyl (\#3 recycle code) }\end{array}$ \\
\hline
\end{tabular}




\section{centennialchallenges}

\section{Phase II Competition: Materials}

\begin{tabular}{|l|l|l|l|c|c|c|c|c|c|c|}
\hline Material Applicability & \multicolumn{10}{|l|}{$\begin{array}{l}\text { Least } \\
\text { Relevant }\end{array}$} \\
\hline $\begin{array}{l}\text { Fine Rock Aggregate } \\
\text { (<1/4" mean particle diameter) }\end{array}$ & & MR & & IRS & & CSR & & GSS & BSR & CBI \\
\hline $\begin{array}{l}\text { Coarse Rock Aggregate } \\
\text { ( } 1 / 4 \text { " mean particle diameter) }\end{array}$ & MR & & IRS & & CSR & & GSS & BSR & CBI & \\
\hline Trash Recyclables & VY & PS & AF & & PP & NY & & PT & HP & LP \\
\hline 3DP Factor & 1 & 2 & 3 & 4 & 5 & 6 & 7 & 8 & 9 & 10 \\
\hline
\end{tabular}

$\mathrm{CBI}$ - Crushed basaltic igneous rock ( $\mathrm{SiO} 2$ weight percent less than or equal to 57 )

BSR - Basaltic sedimentary rocks (talus, alluvium with very little alteration/weathering, or mine tailings)

GSS - Gypsum sand and siliceous sedimentary rocks (e.g., sand box sand, mudstone)

CSR - Carbonaceous sedimentary rocks (e.g., limestone, dolomite)

IRS - Igneous rocks with $\mathrm{SiO} 2$ weight percent greater than 57 (e.g. granite)

LP - LDPE polyethylene (\#4 recycle code)

HP - HDPE polyethylene (\#2 recycle code)

PT - Polyethylene Terephthalate (\#1 recycle code)

NY - Nylon (\#7 recycle code)

PP - Polypropylene (\#5 recycle code)

AF - Aluminum foil or ground up aluminum parts

PS - Polystyrene (\#6 recycle code)

VY - Vinyl (\#3 recycle code)

MR - Metamorphic rocks (e.g., slate) 


\section{centennialchallenges}

\section{Phase II Competition: Level 1}

Specimen 1

- Truncated cone with a tolerance of $+7 \mathrm{~mm}$

- Extruded material must maintain the printed height to within 15\% for a minimum of 5 minutes

Specimen 2

- Compression specimen (300 $\mathrm{mm}$ height and $150 \mathrm{~mm}$ diameter) tested per ASTM C39

- Minimum compressive load $450 \mathrm{~kg}$
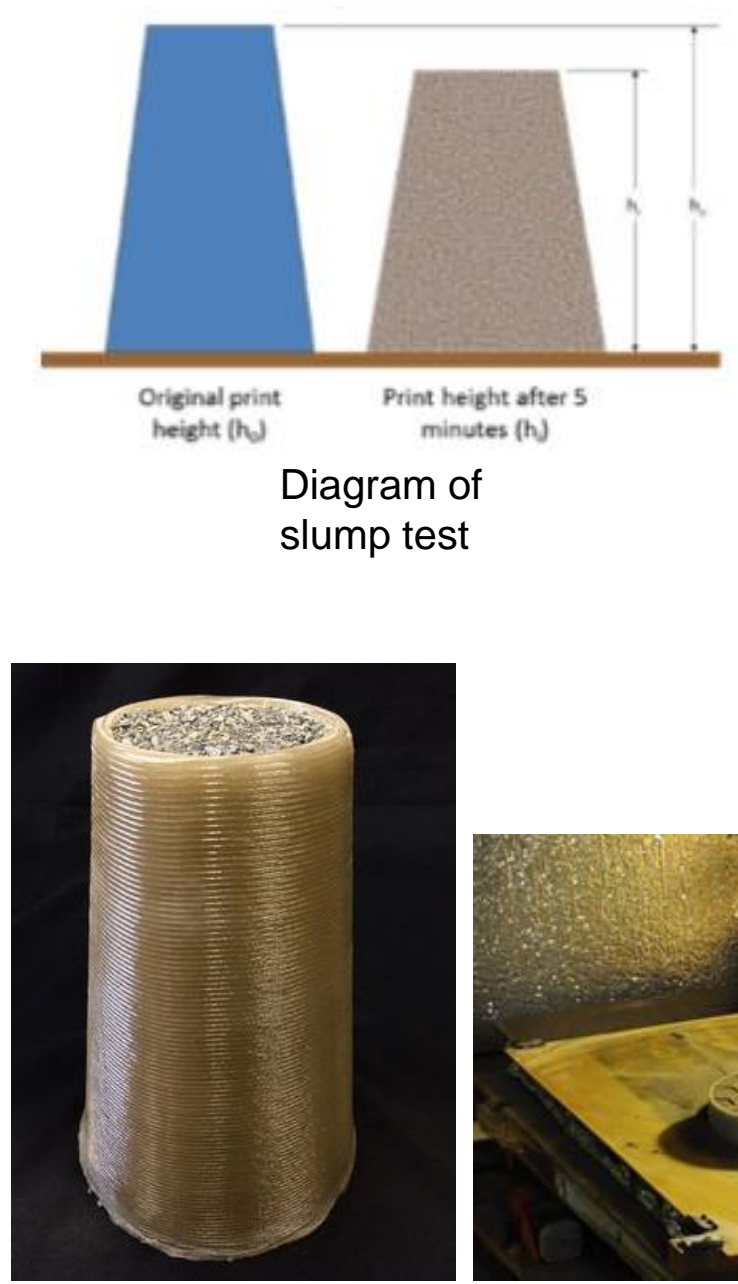

Winning level 1 entry from Foster + Partners and Branch Technology

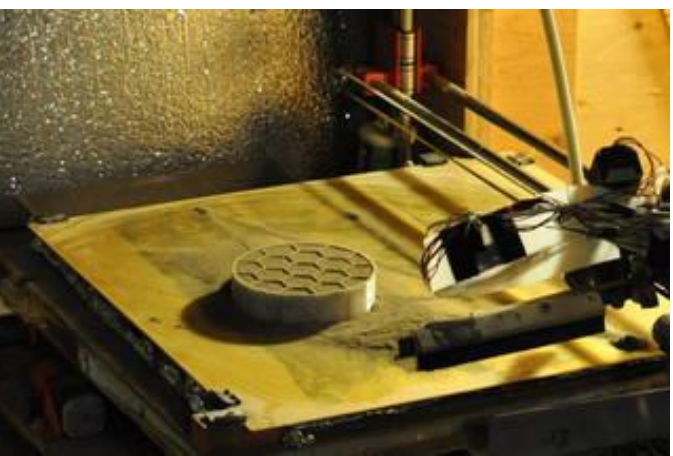

Second place: University of Alaska at Fairbanks 


\section{centennialchallenges}

\section{Phase II Competition: Level 1}

Composite Scoring Equation for Level 1 C39 Cylinder Compression Test

$\left\{\%\right.$ mass $\times 3 D P_{\text {indigenous }}+\%$ mass $\times 3$ pfactor $_{\text {imported }}+\%$ mass $\left.\times 3 D P_{\text {recyclable }}\right\} / 1000$ $\times$ Compressive Cylinder Load + slump test

\begin{tabular}{|l|c|c|c|c|c|}
\hline Level 1 Example Score Calculation & $\begin{array}{c}\text { Actual } \\
\text { Rating }\end{array}$ & Units & 3DP Factor & $\begin{array}{c}\text { Weight } \\
\text { Multipliers }\end{array}$ & $\begin{array}{c}\text { Level 1 } \\
\text { Challenge } \\
\text { Points }\end{array}$ \\
\hline Use of indigenous materials & 80 & $\%$ mass & 5 & 400 & \\
\hline Use of imported materials & 15 & $\%$ mass & -20 & -300 & \\
\hline Use of recyclable materials & 0 & $\%$ mass & 7 & 0 & \\
\hline Use of water & 5 & $\%$ mass & -10 & -50 & \\
\hline $\begin{array}{l}\text { Measured maximum supported mass from the ASTM } \\
\text { C39 compression specimen }\end{array}$ & 454 & kg & & & 0.05 \\
\hline Truncated cone score (0 or 100) & 100 & points & & & 23 \\
\hline Total Points & & & & & 100 \\
\hline
\end{tabular}




\section{centennialchallenges}

\section{Phase II Competition: Level 2}

Specimen

- Beam $60 \mathrm{~cm}$ length $\times 200 \mathrm{~mm}$ height $\times 100 \mathrm{~mm}$ wide cross-section

- Tested per ASTM C78

- Tolerance for specimen width and height was $+7 \mathrm{~mm}$

- Tolerance for length was $+/-7 \mathrm{~mm}$

- $1^{\text {st }}$ place: MoonX (Seoul, South Korea)

- $2^{\text {nd }}$ place: Oregon State University

- $3^{\text {rd }}$ place: Foster+Partners and Branch Technology

- $4^{\text {th }}$ place: University of Alaska, Fairbanks

- $5^{\text {th }}$ place: CTL Group

- $6^{\text {th }}$ place: ROBOCON (Singapore)

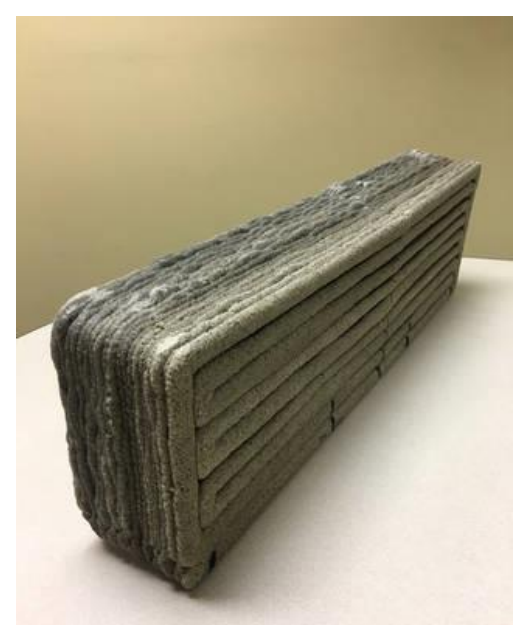

Second-place team Form Forge of Oregon State University, Corvallis, printed this beam for the phase II, level 2 challenge. Image courtesy Form Forge. 


\section{centennialchallenges}

\section{Phase II Competition: Level 2}

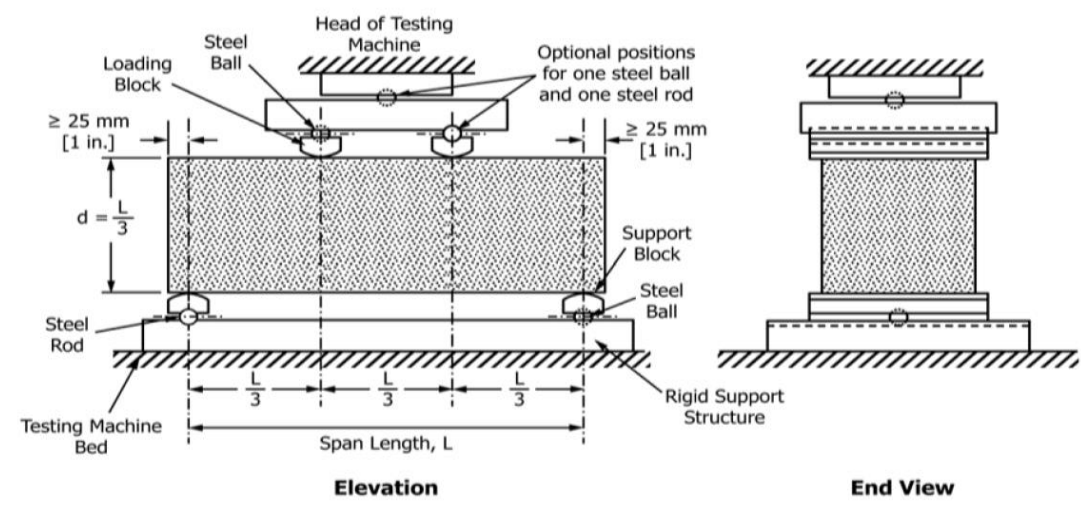

$\frac{\% \text { mass } \times 3 D P_{\text {indig }}+\% \text { mass } \times 3 D P_{\text {imp }}+\% \text { mass } \times 3 D P_{\text {recyc }}+\% \text { mass } \times 3 D P_{\text {water }}}{1000}$ $=D P_{\text {factor }}$

Level 2 Score $=D P_{\text {factor }} \times C 78$

\begin{tabular}{|l|c|c|c|c|c|}
\hline Level 2 Example Score Calculation & \multicolumn{1}{|c|}{$\begin{array}{c}\text { Actual } \\
\text { Rating }\end{array}$} & Units & $\begin{array}{c}\text { 3DP } \\
\text { Factor }\end{array}$ & $\begin{array}{c}\text { Weight } \\
\text { Multipliers }\end{array}$ & $\begin{array}{c}\text { Level 2 } \\
\text { Challenge } \\
\text { Points }\end{array}$ \\
\hline Use of indigenous materials & 80 & $\%$ mass & 5 & 400 & \\
\hline Use of imported materials & 15 & $\%$ mass & -20 & -300 & \\
\hline Use of recyclable materials & 0 & $\%$ mass & 7 & 0 & \\
\hline Use of water & 5 & $\%$ mass & -10 & -50 & \\
\hline $\begin{array}{l}\text { Measured maximum supported mass } \\
\text { from the ASTM C78 flexural } \\
\text { specimen }\end{array}$ & 750 & $\mathrm{~kg}$ & & 0.05 & 38 \\
\hline Total Points & & & & & 38 \\
\hline
\end{tabular}




\section{centennialchallenges}

\section{Phase II Competition: Level 3}

- Head to head competition at Caterpillar's Edwards Demonstration Facility in Peoria, Illinois

- 5 teams invited to Level 3 competition based on successful completion of Level 1 and Level 2

- 3 teams competed from August 23-August 26, 2017

- MoonX (South Korea)

- Foster+Partners and Branch Technology (Chattanooga)

- Penn State

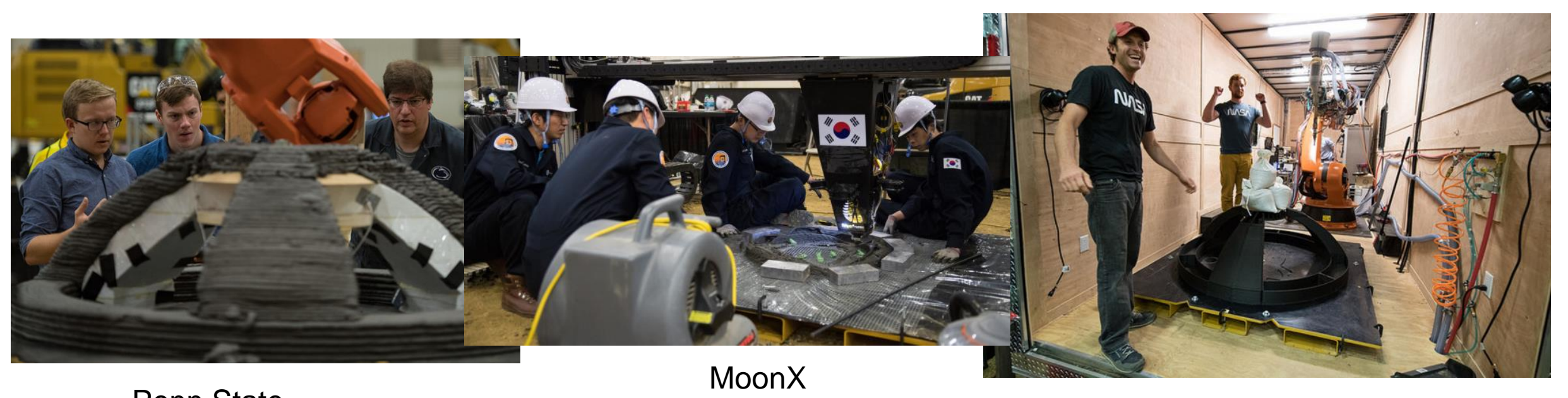




\section{centennialchallenges}

\section{Phase II Competition: Level 3}

- Specimens for Level 3

- Three compression cylinders (300 mm in height x $150 \mathrm{~mm}$ in diameter) printed onsite and tested per ASTM C39

- Three flexure specimens $(60 \mathrm{~cm}$ length $\times 200 \mathrm{~mm}$ height $\times 100 \mathrm{~mm}$ wide cross-section)

- Dome specimen

- Process flow for competition

- Day 1: print cylinders and beams (8 hour printing window)

- Day 2: test cylinders and beams, print dome (12 hour printing window)

- Day 3: test dome

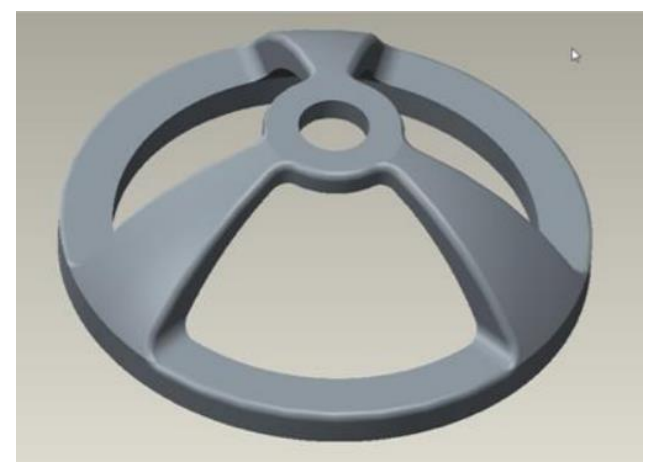

$\frac{\% \text { mass } \times 3 D P_{\text {indig }}+\% \text { mass } \times 3 D P_{\text {imp }}+\% \text { mass } \times 3 D P_{\text {recyc }}+\% \text { mass } \times 3 D P_{\text {water }}}{1000}$ $=D P_{\text {factor }}$

$$
\text { Level } 3 \text { Score }=D P_{\text {factor }} \times\left\{\frac{C 39_{\text {avg }}}{10}+C 78_{\text {avg }}+(\text { Dome } \times 10)\right\}
$$

CAD model of dome 


\section{centennialchallenges}

Phase II Competition: Level 3 Penn State
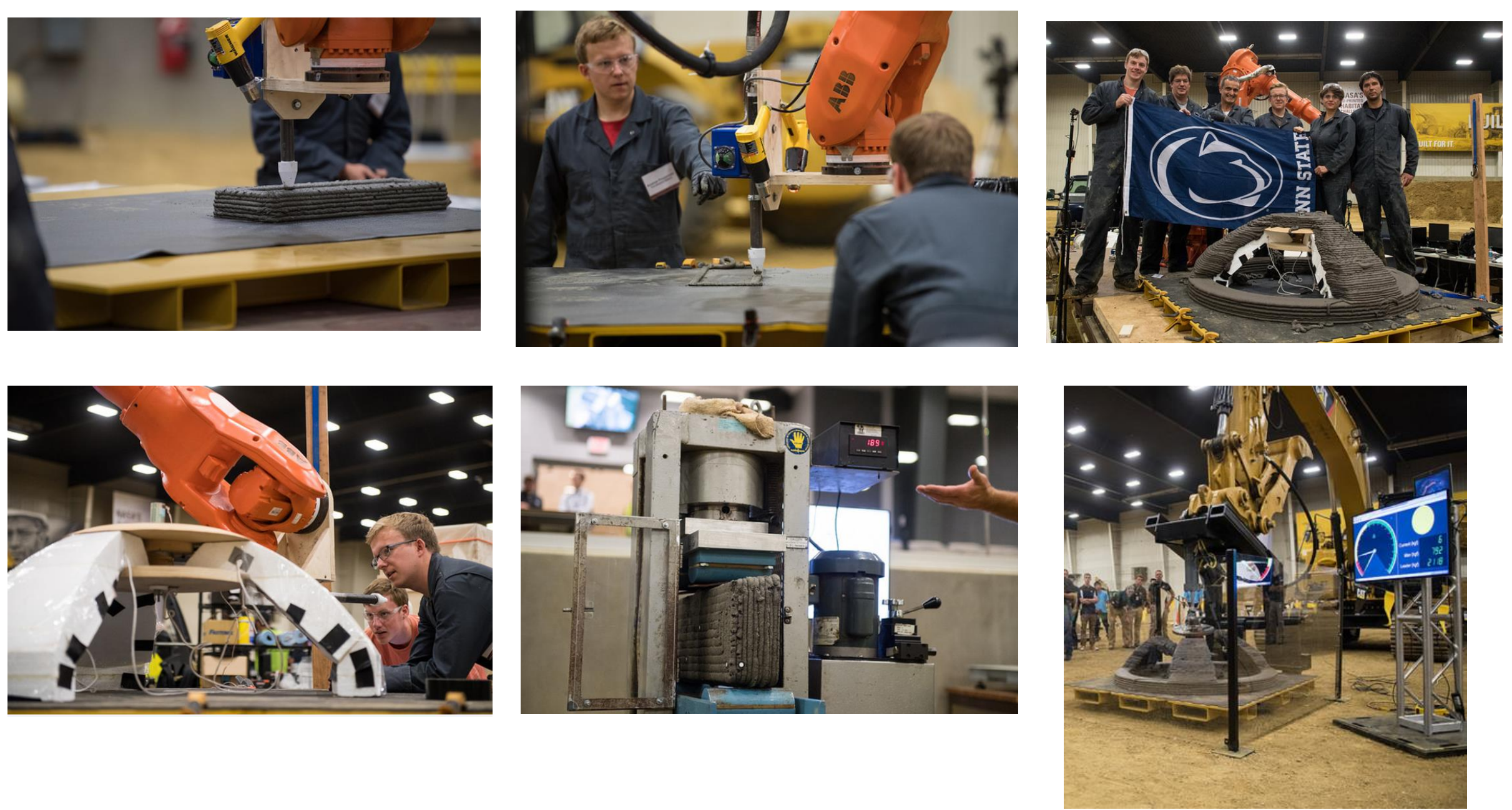


\section{centennialchallenges}

Phase II Competition: Level 3 MoonX
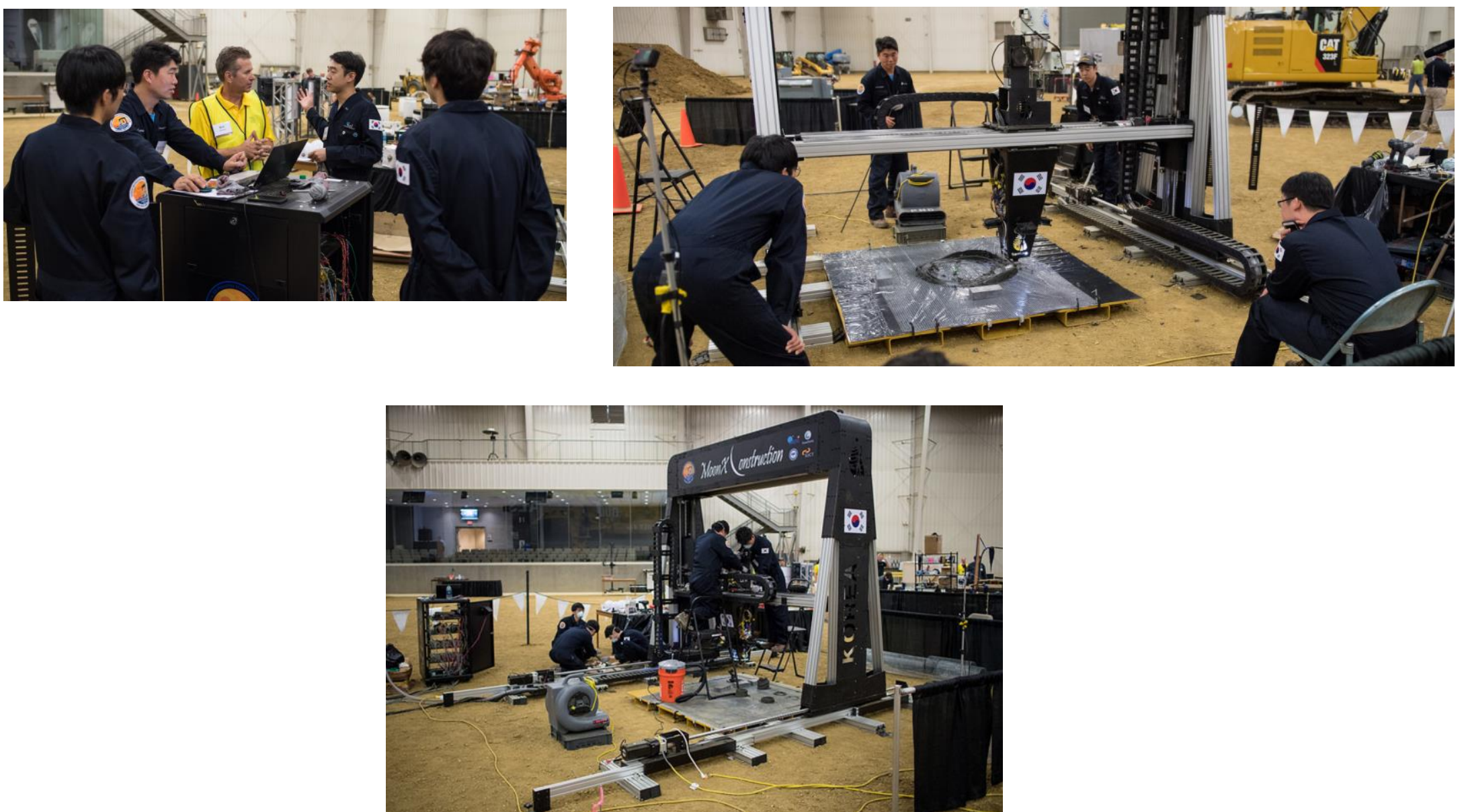


\section{centennialchallenges}

Phase II Competition: Level 3 Foster + Partners and Branch Technology
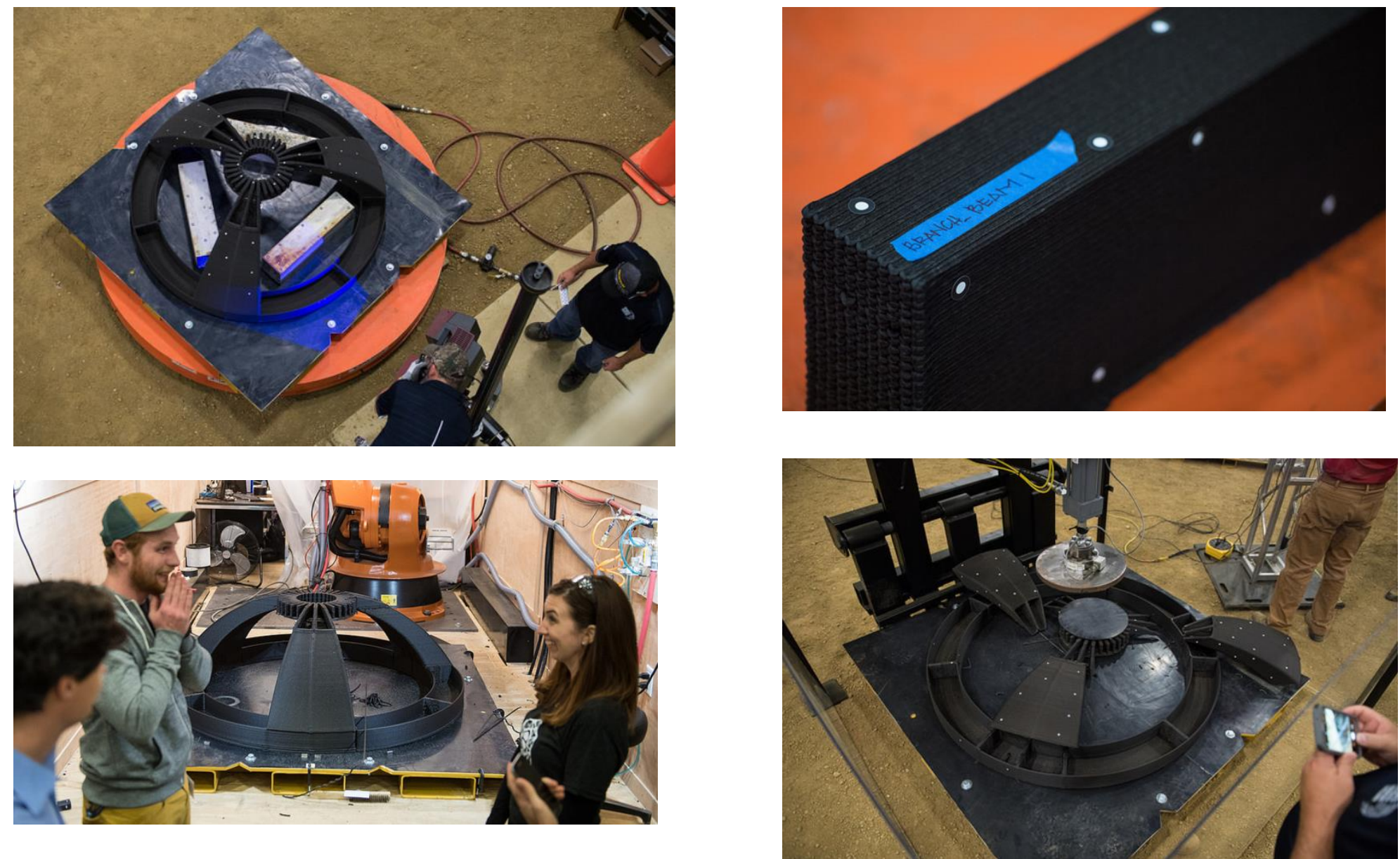

22 


\section{centennialchallenges}

Phase II Competition: Level 3

Results

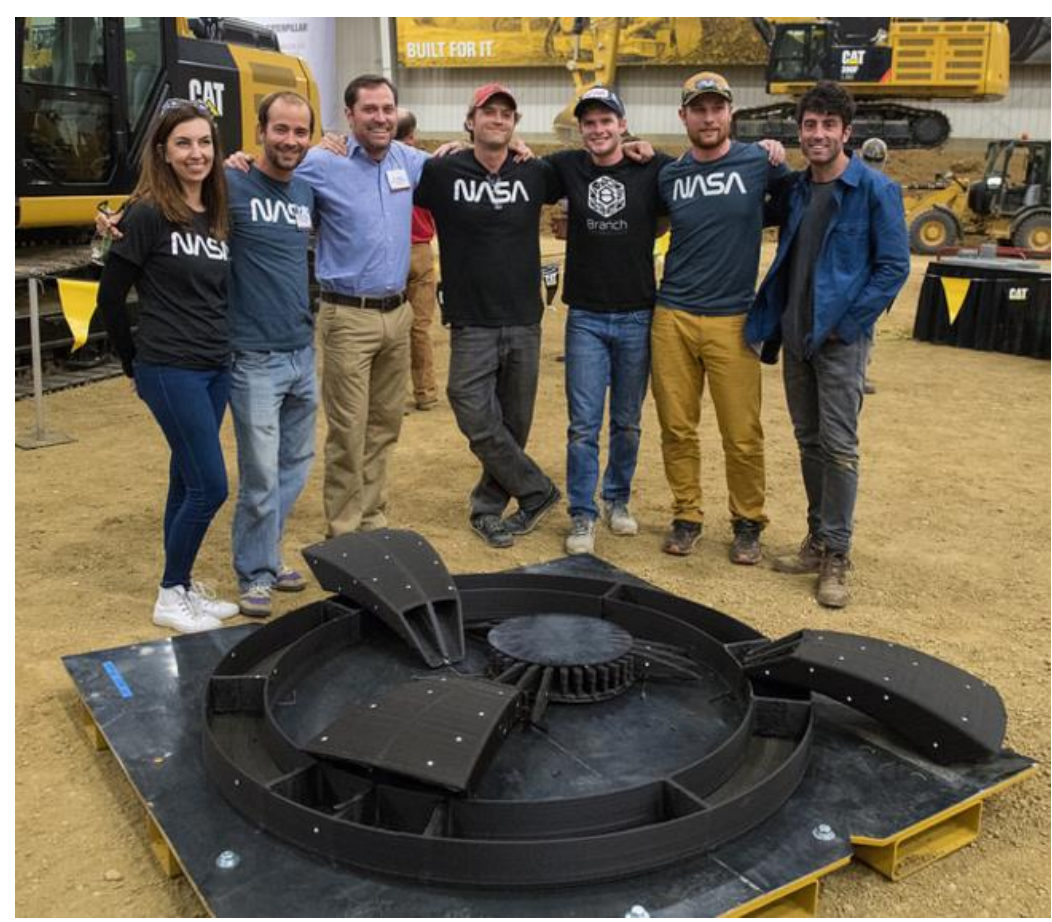

$1^{\text {st }}$ place, $\$ 250,000$ :

Branch Technology and

Foster + Partners

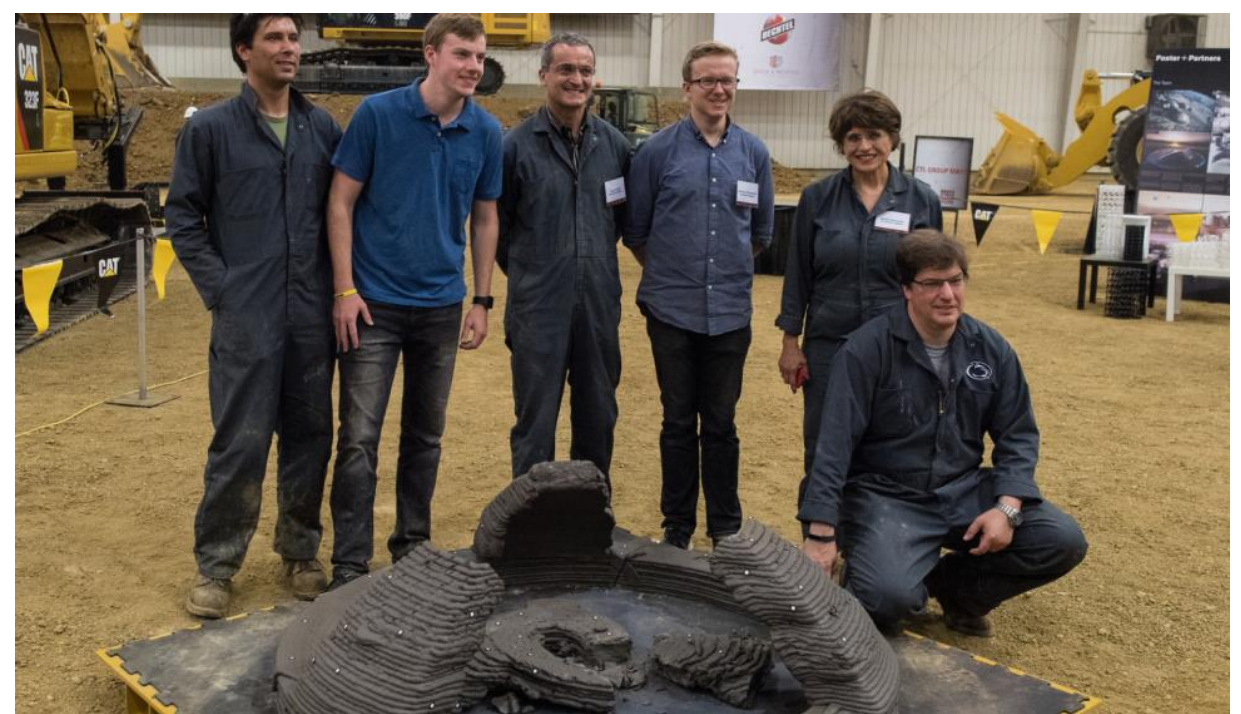

$2^{\text {nd }}$ place, $\$ 150,000$ :

Penn State University 


\section{centennialchallenges}

\section{Significance of the 3D Printed Habitat Challenge}

- Challenge was successful in spurring innovation in the materials, processes, and manufacturing systems needed to manufacture an offworld habitat using mission recycled materials and/or indigenous materials.

- The construction industry is a 3 trillion dollar per year industry and technology advancements made through this challenge may provide beneficial new solutions for revitalizing infrastructure, providing cheaper housing, and enabling improved disaster response.

- Scaleability is the major challenge that will be addressed through the phase III competition.

- 3D-Printed Habitat Challenge Phase 3, Request for Information: www.fbo.gov/index?s=opportunity \&mode $=$ form \& id $=7$ e5f6badeb0c $51 \mathrm{~cd} 8$ $\underline{8 a 65 e a 59789495 f \& t a b=\text { core } \& \quad c v i e w=0}$ 